\title{
Yield Stress Distribution in Injection-Molded Glassy Polymers
}

\author{
Wilco M.H. Verbeeten ${ }^{a, *}$, Marc J.W. Kanters ${ }^{b}$, Tom A.P. Engels ${ }^{b, c}$, \\ Leon E. Govaert ${ }^{b}$ \\ ${ }^{a}$ Structural Integrity Research Group, \\ University of Burgos, Avenida Cantabria s/n, E-09006 Burgos, Spain \\ *E-mail: wverbeeten@ubu.es \\ ${ }^{b}$ Materials Technology (MaTe), Eindhoven University of Technology, \\ P.O. Box 513, NL-5600 MB Eindhoven, the Netherlands \\ ${ }^{c}$ DSM Ahead B.V., Geleen, the Netherlands
}

How to cite: Verbeeten, W. M., Kanters, M. J., Engels, T. A. and Govaert, L. E. (2015), Yield stress distribution in injection-moulded glassy polymers. Polym. Int., 64(11): 1527-1536. doi: 10.1002/pi.4898

February 25, 2015 


\begin{abstract}
A methodology for structural analysis simulations is presented that incorporates the distribution of mechanical properties along the geometrical dimensions of injection molded amorphous polymer products. It is based on a previously developed modeling approach, where the thermo-mechanical history experienced during processing was used to determine the yield stress at the end of an injection molding cycle. Comparison between experimental data and simulation results showed an excellent quantitative agreement, both for short-term tensile tests, as well as long-term creep experiments over a range of strain rates, applied stresses, and testing temperatures. Changes in mold temperature and component wallthickness, which directly affect the cooling profiles and, hence, the mechanical properties, were well captured by the methodology presented. Furthermore, it turns out that the distribution of the yield stress along a tensile bar is one of the triggers for the onset of (strong) localization generally observed in experiments.
\end{abstract}

Keywords: Mechanical properties; modeling; polycarbonate; aging; creep 


\section{Introduction}

Useful service life of a polymer product is mostly determined by its ultimate failure behavior, which can either occur under creep circumstances, due to fatigue, or upon impact. Hence, for a correct and safe polymer product design, the understanding of this failure performance is of utmost importance. The macroscopic deformation and failure behavior of polymers is governed by the onset of plastic strain localization ${ }^{1-3}$. In turn, this is dominated by the large strain intrinsic behavior, i.e. viscoelastic behavior up to yield, followed by strain softening and subsequent strain hardening, as was shown in a quantitative manner by various research groups ${ }^{3-10}$.

A polymer's intrinsic deformation behavior is defined as the material's true strain-stress response applying a globally homogeneous deformation field. In standard mechanical characterization tests, e.g. uniaxial tensile testing, generally strain localization phenomena occur, like necking, shear banding, crazing and cracking, resulting in an inhomogeneous deformation field. Therefore, the measurement of a polymer's intrinsic behavior requires special experimental set-ups, such as "friction-free" uniaxial compression tests ${ }^{8,11}$ or video-controlled tensile tests $^{12,13}$.

However, the intrinsic properties of a polymer product are not constants. They are inhomogeneous along the product's geometry and, additionally, will show a change over time. Both features have their origin in the processing phase and are due to the polymer's state of non-equilibrium at service temperature ${ }^{14,15}$. Polymer products that are melt processed, for example, start off at the (high) melt temperature, where the material is at thermodynamic equilibrium, and are shaped and 
cooled down rapidly to the (low) ambient temperature. During this process, the polymer molecules, which feature relatively long relaxation times, will try to accommodate to the changing conditions, i.e. temperature, pressure, and strain, by molecular re-arrangements. Eventually, molecular chain mobility slows down to such an extent that in the available time-frame it is impossible for the material to relax to its thermodynamic equilibrium. Since there is a drive towards thermodynamic equilibrium, properties are affected over time, a phenomenon called physical aging ${ }^{14,15}$.

Hence, it is rapidly understood that the exact quantitative values of the intrinsic properties are not only determined by the molecular architecture, i.e. the primary chemical structure of the repeating unit. They also depend upon the lamellar thickness ${ }^{16,17}$ and orientation ${ }^{18,19}$ of the crystalline phase (if present) and the thermodynamic state of the amorphous phase ${ }^{10,20}$.

The effect of thermal treatments on the properties of polymer materials has been researched extensively over the years. The changing properties over time and the effect of thermal pre-treatments below the glass temperature have been investigated by Struik ${ }^{14,21}$ and Bauwens-Crowet and Bauwens ${ }^{22}$, respectively. The volume or enthalpy relaxation of glassy materials as well as the yield stress development applying different thermal history profiles from above to below the glass temperature has also been reported, including temperature jumps ${ }^{23}$, continuous heating or cooling ${ }^{24}$, three-step thermal cycles ${ }^{25}$, and temperature profiles resulting from injection molding processing conditions ${ }^{26}$. All studies showed convincingly that different thermal histories result in a distinct non-equilibrium thermodynamic state of the polymer molecules.

In general, the thermo-mechanical history of the different material elements 
along a polymer product is inhomogeneous, especially for the injection molding fabrication process ${ }^{27}$. As a consequence, the thermodynamic (non-equilibrium) state of the material elements will be different at changing locations within a product. In the Eindhoven Glassy Polymer (EGP) model ${ }^{10}$, all variations in thermomechanical history are captured in a single state parameter " $S$ ", which is related to the amount of strain softening during yielding. All other model parameters are independent of thermal history and can be determined separately and uniquely from the polymer's large strain intrinsic behavior. The model accounts for deformation kinetics and aging kinetics and it was shown to quantitatively predict short- and long-term failure, both static and dynamic ${ }^{28,29}$.

In previous studies by Govaert and coworkers ${ }^{26,27,30,31}$, a methodology was developed that allows for a direct and quantitative prediction of the development of yield stresses during processing of glassy polymers. For this method, standard polymer processing simulation tools were employed to determine the transient thermal history that follows from cooling from the melt in each material element of a molded product. By combining that information with the aging kinetics, a yield stress distribution throughout the complete polymer product was calculated. Computed yield stresses of injection-molded plates proved to be in good agreement with the experimentally obtained values.

To enable comparison of the numerical predictions with experimentally determined yield stresses, a thickness-weighted average of the computed yield stress distribution was used in all structural analysis simulations ${ }^{26,27,30}$. That is, all elements of the finite element mesh were given the same value for the non-equilibrium thermodynamic state. Although it resulted in a quantitative agreement between experiments and numerical predictions, it does not allow designers to gain insight 
into the consequences of specific choices of e.g. the location of cooling channels in the mold on the service life performance of complex-shaped products.

For most of the experimentally obtained yield stresses in these studies, rectangular bars were cut from injection molded rectangular plates, which had the need for a post-processing machining operation to obtain a gauge section. These machining operations are believed to influence the thermodynamic state of the surface, thus influencing experimental results ${ }^{30}$. Although this effect can be minimized with proper precautions, it is never completely eliminated.

In the present study, we will focus on a methodology for structural analysis simulations, that incorporates the distribution of yield stresses along the geometrical dimensions of an injection molded amorphous polymer product. The beforementioned methodology 27,30 is adopted, where in the present study we make use of previously published data for the same material, but different grades, to determine the deformation and aging kinetics. Contrary to the previous studies, here the calculated yield stress distribution along the product is used for the structural analysis simulations instead of an average value for the whole product. An improved agreement between experimental results and structural analysis simulations could be achieved. Furthermore, it turns out that the distribution of intrinsic properties is one of the triggers for the onset of the (strong) localization generally observed in experiments. From an engineering point of view, this methodology can provide a powerful tool for polymer product and mold designers to understand upfront the consequences of their choices on service life in a very early design stage. 


\section{Modeling}

\section{Constitutive Equation}

As a constitutive model for the material behavior, the multi-mode $3 \mathrm{D}$ elastoviscoplastic Eindhoven Glassy Polymer (EGP) model ${ }^{10,32,33}$ is used, known to accurately capture the intrinsic deformation characteristics of polymeric glasses. The basis of the EGP-model is the split of the Cauchy stress into a hardening stress, $\boldsymbol{\sigma}_{r}$, and a driving stress, $\boldsymbol{\sigma}_{s}$, first proposed by Haward and Thackray ${ }^{34}$ :

$$
\boldsymbol{\sigma}=\boldsymbol{\sigma}_{r}+\boldsymbol{\sigma}_{s}
$$

The strain hardening contribution is attributed to the molecular orientation of the entangled polymer network and is modeled by a Neo-Hookean elastic expression:

$$
\boldsymbol{\sigma}_{r}=G_{r} \tilde{\boldsymbol{B}}^{d}
$$

consisting of the strain-hardening modulus $G_{r}$ and the deviatoric part of the isochoric left Cauchy-Green strain tensor $\tilde{\boldsymbol{B}}$. The driving stress, attributed to intermolecular interactions on a segmental scale, is decomposed into a hydrostatic part and a deviatoric part, representing the volumetric change and the rate-dependent plastic flow, respectively. The deviatoric driving stress is modeled by a non-linear multi-mode Maxwell model ${ }^{32,33}$ :

$$
\boldsymbol{\sigma}_{s}=\boldsymbol{\sigma}_{s}^{h}+\boldsymbol{\sigma}_{s}^{d}=\kappa(J-1) \boldsymbol{I}+\sum_{i=1}^{n} G_{i} \tilde{\boldsymbol{B}}_{e, i}^{d}
$$


Here, $\kappa$ is the bulk modulus, $J$ the volume change ratio, $I$ the unity tensor, and $G$ is a shear modulus. The evolution equations of the volumetric and elastic strains of the different modes are given by:

$$
\begin{gathered}
\dot{J}=J \operatorname{tr}(\boldsymbol{D}), \\
\dot{\tilde{\boldsymbol{B}}}_{e, i}=\left(\tilde{\boldsymbol{L}}-\boldsymbol{D}_{p, i}\right) \cdot \tilde{\boldsymbol{B}}_{e, i}+\tilde{\boldsymbol{B}}_{e, i} \cdot\left(\tilde{\boldsymbol{L}}^{c}-\boldsymbol{D}_{p, i}\right),
\end{gathered}
$$

where $\tilde{\boldsymbol{L}}$ is the isochoric velocity gradient tensor, and $\boldsymbol{D}_{p}$ is the plastic part of the rate of deformation tensor $D$, defined as:

$$
\boldsymbol{D}_{p, i}=\frac{\boldsymbol{\sigma}_{s, i}^{d}}{2 \eta_{i}(\bar{\tau}, p, S)} .
$$

The viscosities $\eta_{i}$ are based on an Eyring equation, modified to take pressure and intrinsic strain softening into account:

$$
\eta_{i}=\eta_{0, i, r e f} \exp \left(\frac{\Delta U_{y}}{R}\left[\frac{1}{T}-\frac{1}{T_{r e f}}\right]\right) \frac{\bar{\tau} / \tau_{0}}{\sinh \left(\bar{\tau} / \tau_{0}\right)} \exp \left(\frac{\mu p}{\tau_{0}}\right) \exp (S)
$$

where $\eta_{0, i, r e f}$ is the zero viscosity of the $i^{\text {th }}$ relaxation mode in the rejuvenated state at the reference temperature, $\Delta U_{y}$ the activation energy for the temperature dependence, $R$ the gas constant, $T_{r e f}$ the reference temperature, $\bar{\tau}$ is the total equivalent stress, $\tau_{0}$ is a characteristic stress, and $S$ is the thermodynamic state parameter that captures the effect of physical aging and strain softening. The total equivalent stress, the characteristic stress, and the hydrostatic pressure are defined as:

$$
\bar{\tau}=\sqrt{\frac{1}{2} \boldsymbol{\sigma}_{s}^{d}: \boldsymbol{\sigma}_{s}^{d}} \quad, \quad \tau_{0}=\frac{R T}{V_{y}^{*}} \quad, \quad p=-\frac{1}{3} \operatorname{tr}\left(\boldsymbol{\sigma}_{s}\right),
$$


where $V_{y}^{*}$ is the activation volume.

The thermodynamic state parameter $S$ is composed of two contributions, which are assumed to be fully decoupled:

$$
S\left(t, T, \bar{\gamma}_{p}\right)=S_{a}(t, T) \cdot R\left(\bar{\gamma}_{p}\right)
$$

where $S_{a}$ represents the aging kinetics depending on time and temperature, and $R\left(\bar{\gamma}_{p}\right)$ is the softening function as a result of mechanical rejuvenation determined by the equivalent plastic strain $\bar{\gamma}_{p}$. For short-term experiments, the state parameter $S_{a}$ is usually a constant indicating the initial thermodynamic state of the material. The evolution of this state parameter $S_{a}$ will be described in more detail in the next subsections. The softening function $R\left(\bar{\gamma}_{p}\right)$ is described using a modified CarreauYasuda expression that is normalized to 1 and decreases monotonically to 0 with increasing (equivalent) plastic strain:

$$
R\left(\bar{\gamma}_{p}\right)=\frac{\left(1+\left[r_{0} \cdot \exp \left(\bar{\gamma}_{p}\right)\right]^{r_{1}}\right)^{\left(r_{2}-1\right) / r_{1}}}{\left(1+r_{0}^{r_{1}}\right)^{\left(r_{2}-1\right) / r_{1}}},
$$

where $r_{0}, r_{1}$, and $r_{2}$ are fitting parameters. The plastic strain evolution is coupled to the longest relaxation time, i.e. mode $i=1$, and defined as:

$$
\dot{\bar{\gamma}}_{p}=\frac{\bar{\tau}_{1}}{\eta_{1}}
$$

where the equivalent stress of the longest relaxation time is $\bar{\tau}_{1}=\sqrt{\frac{1}{2} \boldsymbol{\sigma}_{s, 1}^{d}: \boldsymbol{\sigma}_{s, 1}^{d}}$.

Model parameters and the multi-mode relaxation spectrum for amorphous polycarbonate are adopted from literature ${ }^{10,33,35}$ and are given in the Tables 1 and 
2. These parameters are determined from "friction-free" uniaxial compression ${ }^{10,33}$ and uniaxial tensile tests ${ }^{35}$ at different strain rates and temperatures and turned out to be valid for different grades of polycarbonate, irrespective of the material's molecular weight ${ }^{10}$. The only parameter which still has to be determined as a function of the initial thermodynamic state of the material, is the $S_{a}$-parameter. The response of the EGP model is shown in Figure 1, where previously published experimental data ${ }^{10,33}$ (symbols) are compared to model predictions (solid lines), showing an excellent agreement between experiments and predictions. Figure 1(a) shows the model response for polycarbonate in the same thermodynamic state, represented by $S_{a}=27.5$, at different strain rates. Figure 1(b) shows the model response for polycarbonate with different thermal histories at a single strain rate of $10^{-3} \mathrm{~s}^{-1}$, represented by $S_{a}=27.5$ for quenched polycarbonate and by $S_{a}=30.3$ for annealed polycarbonate.

\section{Aging Kinetics}

In short-time experiments, the aging kinetics parameter $S_{a}$ is usually a constant. However, for long-term experiments or experiments at elevated temperatures, the yield stress starts to increase due to physical aging. It was shown by Klompen et $a l .{ }^{10}$, that the aging kinetics could be conveniently captured by a change of the yield stress as a function of an effective aging time and temperature (see Figure 2(a)). These results could be combined into a single master curve using annealing time-temperature superposition ${ }^{10}$ (see Figure 2(b)). Since the state parameter $S_{a}$ is directly proportional to the yield stress, an evolution equation could 


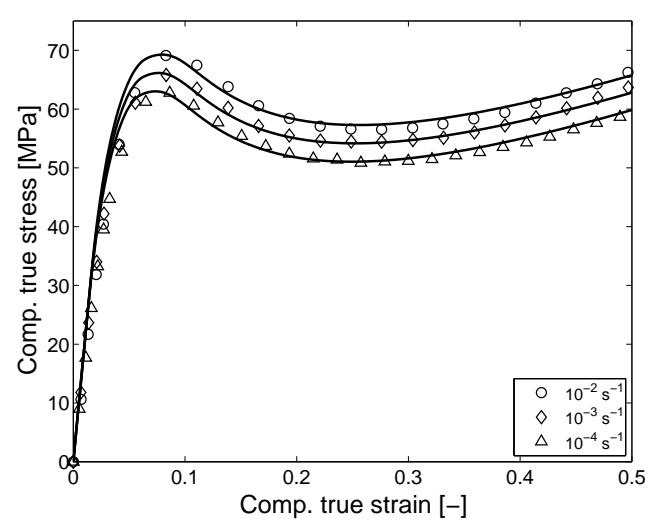

(a)

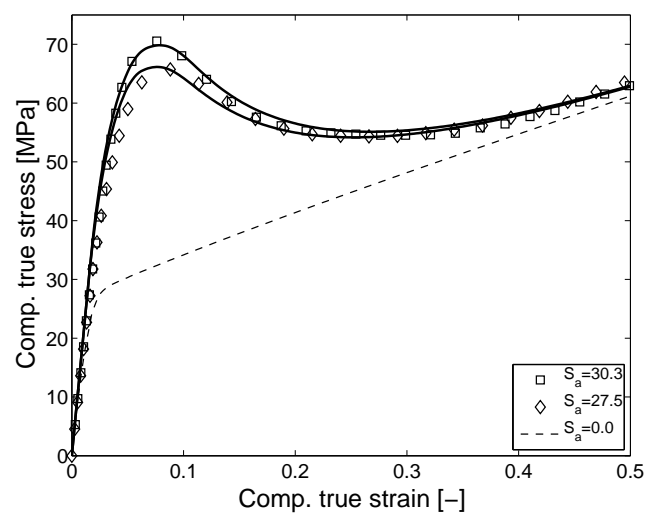

(b)

Figure 1: Intrinsic stress-strain response of polycarbonate in uniaxial compression tests. (a) Stress-strain response at different strain rates and an initial thermodynamic state of $S_{a}=27.5$; $(b)$ Stress-strain response with different thermal histories (i.e. quenched and annealed) at a strain rate of $10^{-3} \mathrm{~s}^{-1}$. Symbols are experimental data; solid lines are model predictions; dashed line is the completely rejuvenated state. Experimental data are taken from Klompen et al. ${ }^{10}$ and Van Breemen et al. ${ }^{33}$.

be determined from the master curve ${ }^{10}$, that is given by:

$$
S_{a}(t, T)=c_{0}+c_{1} \log \left(\frac{t_{e f f}(t, T)+t_{a}}{t_{0}}\right)
$$

where $c_{0}$ is a constant derived from the intersection of the curve with the vertical axis at $t=1 \mathrm{~s}, c_{1}$ is a constant determined by the slope of the curve, $t_{a}$ is the initial age of the material, $t_{0}=1 \mathrm{~s}$ and $t_{e f f}$ is the effective time.

From the evolution of the yield stress with time, it can be seen that there is an initial height of a constant yield stress, before it starts to increase at a constant rate of increase with logarithmic time (see Figure 2). This initial height of the yield stress is completely determined by the processing conditions and can be defined as the initial age $t_{a}$ of the material ${ }^{10,27}$. Once the initial age $t_{a}$ has passed, the yield 


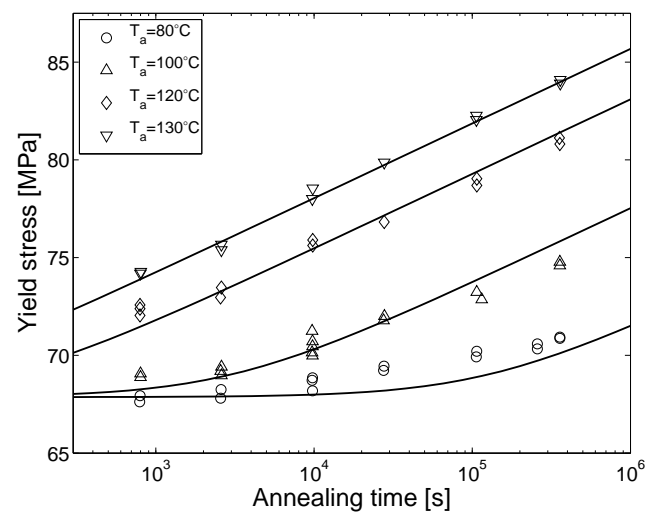

(a)

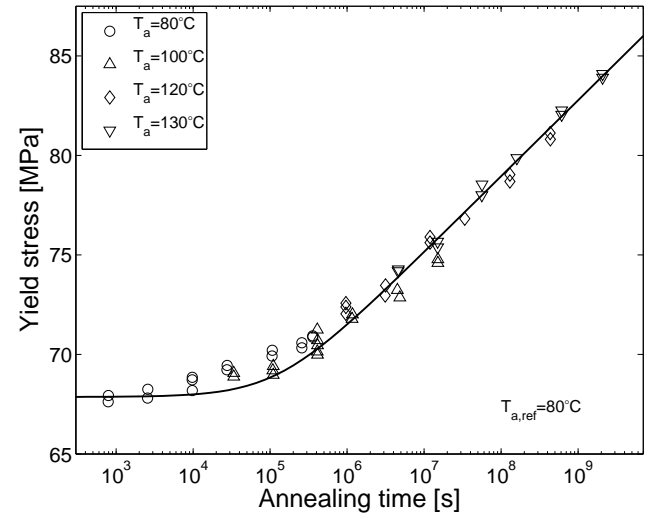

(b)

Figure 2: (a) Evolution of the uniaxial tensile yield stress at a strain rate of $10^{-2} \mathrm{~s}^{-1}$ as a function of annealing time for different annealing temperatures. $(b)$ Master curve for a reference annealing temperature of $80^{\circ} \mathrm{C}$ constructed from the experimental data by using time-temperature superposition. Symbols are experimental data; solid lines are model predictions. Experimental data are taken from Klompen et al. ${ }^{10}$.

stress starts to increase at a rate that is independent of the processing conditions. This evolution is captured by the effective time, defined as:

$$
t_{e f f}(t, T)=\int_{0}^{t} a_{T}^{-1}\left(T\left(t^{\prime}\right)\right) d t^{\prime}
$$

where $a_{T}$ is the time-temperature shift function, described by:

$$
a_{T}(T(t))=\exp \left(\frac{\Delta U_{a}}{R}\left[\frac{1}{T(t)}-\frac{1}{T_{r e f}}\right]\right) .
$$

Here, $\Delta U_{a}$ is the activation energy for aging. A good description of the experimental uniaxial tensile yield stress evolution due to aging is obtained by using the parameters of Table 3 and $t_{a}=8.5731 \times 10^{10} \mathrm{~s}$ at $T_{\text {ref }}=23^{\circ} \mathrm{C}$, as can be seen in Figure 2. 


\section{Processing-Induced Properties}

Thus, $t_{a}$ determines the thermodynamic state of the material after processing, which can be determined by a single tensile test ${ }^{10}$. However, previous studies by Govaert and coworkers ${ }^{26,27,30,31}$ showed that this value can even be predicted by taking into account the thermal history during the processing phase. To predict the evolution of the thermodynamic state of the material during processing, two major assumptions were adopted that proved to give quantitative results: (i) above the glass transition temperature the material is in thermodynamic equilibrium and the effective aging time equals zero; (ii) the physical processes involved in the evolution of the thermodynamic state are identical to those governing the increase in yield stress during annealing below the glass transition temperature $T_{g}$. Thus, following the previously-developed method ${ }^{27}$ by applying Equation (13) to the thermal history a material element experiences during the processing phase, the evolution equation during processing is specified as:

$$
\begin{array}{cc}
T>T_{g}: & t_{e f f, c}=0 \quad \text { and } \quad \dot{t}_{e f f, c}=0, \\
T<T_{g}: & t_{e f f, c}(t, T)=\int_{0}^{t_{c}} a_{T}^{-1}\left(T_{c}\left(t^{\prime}\right)\right) d t^{\prime},
\end{array}
$$

where $t_{e f f, c}$ is the effective aging time which accumulates during processing and that will be equal to the initial age $t_{a}$ of Equation (12) at the end of the processing phase, and $T_{c}(t)$ is the thermal history a material element experiences during the processing phase. From $t_{e f f, c}$, the initial value of the state parameter $S_{a}$ for every material element can be calculated according to Equation (12) and used as an input variable for structural analysis simulations. 
It should be noted that $T_{g}$ is introduced here as an additional constant parameter of value $T_{g}=152.5^{\circ} \mathrm{C}=425.5 \mathrm{~K}$, as determined from DMTA measurements. Obviously, this does not account for the complex dynamics around the glass transition $^{15,36}$. However, previous studies showed that a more physically accurate approach $^{26}$ gave equivalent agreement with experimental data as the more engineering approach ${ }^{27}$. In the present study, in order to reduce computational costs, the more simplified engineering approach is adopted.

\section{Materials and Methods}

\section{Polycarbonate Sample Preparation}

The material used in the present study is a commercial injection molding grade of polycarbonate, PC Lexan 141R (Sabic Innovative Plastics, Bergen op Zoom, the Netherlands; Sabic Innovative Plastics España, Sant Just Desvern, Spain). The number-averaged molecular weight and weight-averaged molecular weight are $9.2 \mathrm{~kg} / \mathrm{mol}$ and $25.8 \mathrm{~kg} / \mathrm{mol}$, respectively. Before any processing, the polycarbonate granules were dried in a vacuum oven at $80{ }^{\circ} \mathrm{C}$ during at least 24 hours.

Tensile bars according to the ASTM D638 Type I test specimen specifications (see Figure 3(a)), with a cross-sectional area of $3.2 \times 13.13 \mathrm{~mm}^{2}$, were injection molded on an Arburg 320S/all-rounder 500-150 injection molding machine, using an Axxicon mold (Axxicon Moulds B.V., Helmond, the Netherlands). The melt temperature was set to $285^{\circ} \mathrm{C}$ and a flow rate of $20 \mathrm{~cm}^{3} / \mathrm{s}$ was used for mold filling of all samples. To ensure a better control of the cooling process and impose the same cooling history for both experiments and simulations, tensile bars were kept 


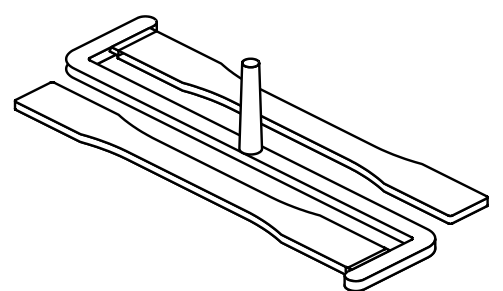

(a)

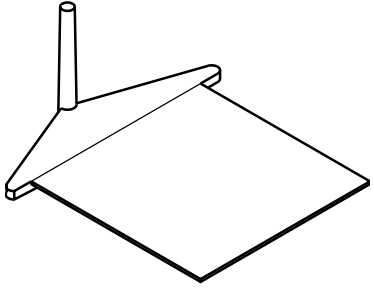

(b)

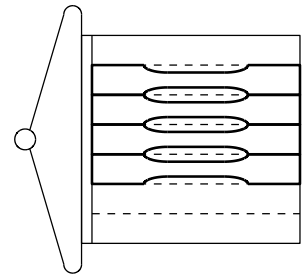

(c)

Figure 3: Injection molded ASTM tensile bars $(a)$, plate $(b)$, and tensile bars made thereof $(c)$.

in the mold until the complete sample was cooled down to the mold temperature. Injection molding simulations demonstrated that a cooling time of $150 \mathrm{~s}$ was sufficient for the highest mold temperature. This cooling time, which is rather long for a standard injection molding cycle, was kept constant for all injection molding batches. Since variation of the holding pressure proved to be of no influence on the measured properties, a three-point holding pressure profile was applied with reference pressures of $750 / 500 / 25$ bar at reference times of $0.1 / 5 / 8$ seconds to minimize shrinkage and flash. Since mold temperature influences the cooling rate and, as a consequence, determines final properties of an injection molding product ${ }^{27}$, the mold cavity temperature was varied from $30{ }^{\circ} \mathrm{C}$ to $130{ }^{\circ} \mathrm{C}$ in steps of $10{ }^{\circ} \mathrm{C}$. After several initial shots, the actual mold temperature was measured and used as an input parameter for the injection molding simulations.

On the other hand, rectangular plates (see Figure 3(b)) with dimensions $70 \times$ $70 \times 1 \mathrm{~mm}^{3}, 70 \times 70 \times 2 \mathrm{~mm}^{3}, 70 \times 70 \times 3 \mathrm{~mm}^{3}$, and $70 \times 70 \times 4 \mathrm{~mm}^{3}$ were injection molded on the same injection molding machine. The same Axxicon mold, but with different inserts, was used for these samples. The melt temperature, mold temperature, and holding pressure were set constant to $285^{\circ} \mathrm{C}, 90^{\circ} \mathrm{C}$, and $500 \mathrm{bar}$, 
respectively. The mold temperature was chosen for it to lie within the processing range recommended by the manufacturer, i.e. between $80{ }^{\circ} \mathrm{C}$ and $120^{\circ} \mathrm{C}$. Flow rates and cooling times for the $1 \mathrm{~mm}, 2 \mathrm{~mm}, 3 \mathrm{~mm}$, and $4 \mathrm{~mm}$ thick plates were set to $90 \mathrm{~cm}^{3} / \mathrm{s}$ and $60 \mathrm{~s}, 50 \mathrm{~cm}^{3} / \mathrm{s}$ and $90 \mathrm{~s}, 50 \mathrm{~cm}^{3} / \mathrm{s}$ and $120 \mathrm{~s}$, and $50 \mathrm{~cm}^{3} / \mathrm{s}$ and 150 s, respectively.

From these plates, bars with dimensions $70 \times 10 \mathrm{~mm}^{2}$ and the various thicknesses were cut parallel to the flow directions and subsequently foreseen with a gauge section of $33 \times 5 \mathrm{~mm}^{2}$ by a precision machining operation, as indicated in Figure 3(c).

\section{Experimental Method}

Uniaxial tensile tests were performed on a Zwick Z010 universal test machine at three different temperatures: $23^{\circ} \mathrm{C}, 40{ }^{\circ} \mathrm{C}$, and $60{ }^{\circ} \mathrm{C}$. For the tests above room temperature, a temperature chamber was used. Samples were freely mounted in the set-up and left for approximately 12 minutes to thermally equilibrate before tightening the clamps. After an additional 5 minutes, the samples were tested. Experiments were performed by applying constant linear strain rates $\left(\dot{\varepsilon}=\dot{x} / l_{0}\right)$. The tensile stresses listed in the results section are engineering stresses $\left(\sigma=F / A_{0}\right)$, and taken as the mean value of at least three experiments. Engineering yield stresses were taken as the maximum stress of the engineering stress-strain curves, just prior to the occurrence of sample necking.

Uniaxial tensile creep tests were performed in the same manner as described above, applying constant engineering stresses $\left(\sigma=F / A_{0}\right)$. Single measurements were performed for each test condition. Engineering strain as a function of time 
was recorded.

\section{Numerical Method}

The thermal history during processing is determined from numerical simulations of the injection molding process, performing fully $3 \mathrm{D}$ computations with the commercial finite element simulation package Autodesk Moldflow Insight (release 2013). Processing parameters were used as mentioned before.

Mesh refinement towards the mold cavity walls was applied in order to capture variations in the temperature cooling profile with sufficient precision. For both the plates and the ASTM samples, all meshes had twelve elements over the thickness direction. The ASTM mold, the $1 \mathrm{~mm}$ plate mold, the $2 \mathrm{~mm}$ plate mold,the $3 \mathrm{~mm}$ plate mold, and the $4 \mathrm{~mm}$ plate mold cavities were meshed with respectively 721647,492 422, 535770,498041 , and 506665 linear tetrahedral elements.

For every node of the meshes used for the injection molding simulations, the time-temperature profile $T(t)$ was extracted. These profiles were then used to calculate the initial state parameter values at the end of the processing step, applying Equation (12), (14), (15), and (16). Calculations were performed by means of a specifically programmed subroutine.

The structural analysis simulations were performed using the commercial finite element package MSC.Marc. The constitutive EGP model is implemented by means of the HYPELA2 user subroutine, in such a way that it can handle different state parameter values for every node in the mesh. Only the part between the test set-up clamps was meshed and analyzed in the simulations. The uniaxial test samples are meshed in full 3D with respectively 9590 , and 9952 linear brick 
elements. Again, mesh refinement towards the upper and lower cavity walls was applied. Since the nodes of the structural analysis meshes do not coincide with the injection molding meshes, nodal $S_{a}$-values are transferred from one mesh to the other by interpolation using a Delaunay triangulation method.

\section{Results and Discussion}

\section{Influence of Cooling Phase}

The cooling profiles of material elements over the height (i.e. $z=0-3.2 \mathrm{~mm}$ ) in the center of an ASTM tensile bar for a mold temperature of $90{ }^{\circ} \mathrm{C}$, as obtained from the numerical simulations, is shown in Figure 4(a). As expected, cooling is faster near the surface and slower at the center of the sample. Two temperature upswings can be seen, which are more pronounced for the profiles towards the mold surface. The first one is around $0.4 \mathrm{~s}$ and is related to the viscous heating of the material at the end of the filling stage. The second temperature upswing around $9 \mathrm{~s}$ is due to the heat balance between the hot melt and the cool mold wall. Similar profiles are seen for other mold temperatures (not shown here).

Figure 4(b) demonstrates the same profiles, but now from the moment that these profiles drop below $T_{g}$. Since that moment is considered as the time that aging begins, that time is redefined as $t=0 \mathrm{~s}$. As a consequence, the cooling profiles shift to the left. A curious result is that, as demonstrated by Figure 4(b), the shifted cooling curves below the (constant) glass transition temperature can be intersecting each other. Of course, it should be taken into account that a constant $T_{g}$ is physically not correct. It is repeatedly demonstrated experimentally, that a 


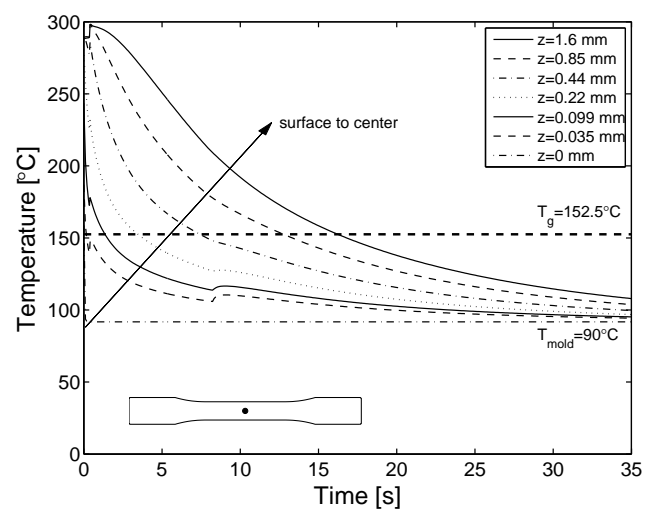

(a)

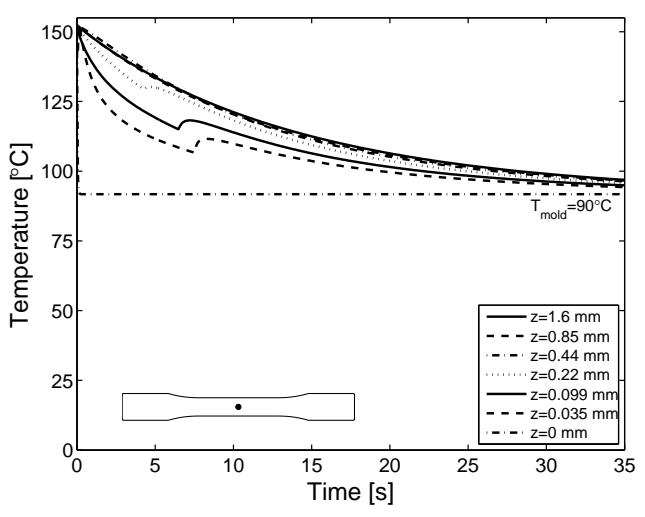

(b)

Figure 4: $(a)$ : Cooling profiles over the height at the center of the injection molded ASTM tensile bar with $T_{\text {mold }}=90{ }^{\circ} \mathrm{C}$. Horizontal dashed line: glass transition temperature $T_{g}$. $(b)$ : Temperature versus time of these profiles from the moment the temperature drops below $T_{g}$.

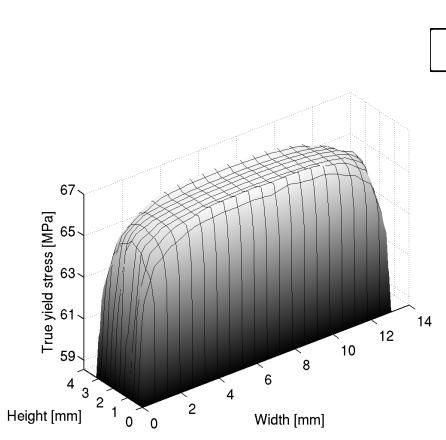

(a)

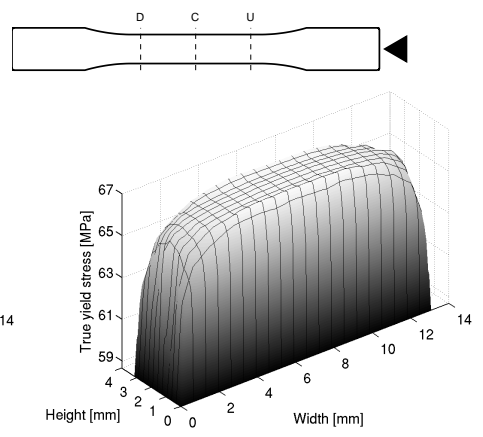

(b)

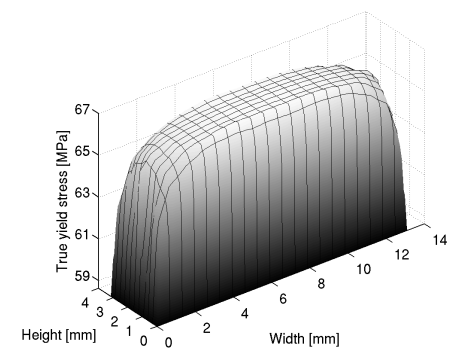

(c)

Figure 5: Predicted true yield stress distribution at $\dot{\varepsilon}=10^{-3} \mathrm{~s}^{-1}$ for a mold temperature of $T_{\text {mold }}=90^{\circ} \mathrm{C}:(a)$ cross-section downstream from the injection point, (b) cross-section at the center of the tensile bar, and (c) cross-section upstream from the injection point.

slower cooling process results in a lower $T_{g}$. Hence, the curve crossover effect shown in Figure 4(b) should be interpreted very cautiously. Nonetheless, the data as shown in Figure 4(b) are used to calculate $S_{a}$ with Equation (16). 
The $S_{a}$-values over the complete ASTM tensile bar were calculated, as well as the related true yield stresses for constant strain rate tensile experiments at $\dot{\varepsilon}=10^{-3} \mathrm{~s}^{-1}$. The results at three different tensile bar cross-sections, i.e. upstream, at the center, and downstream of the injection point, are shown in Figure 5. Values near and at the mold surface cavity are much lower than in the center of the cross-section, where cooling rates are significantly lower. Related to the intersecting temperature profiles, yield stress values in between the center point and the upper and lower mold cavity walls are somewhat higher than in the complete center of the cross-sections. This is due to the fact that at these positions, the temperature can be closer to, but below, (the constant) $T_{g}$ for a longer period of time than for positions at the center of the cross-section, resulting in the development of a higher $S_{a}$-value. Again, these results should be interpreted with care, due to the constant $T_{g}$-value taken for the numerical method presented here. As can be seen, the yield stress distributions for the three cross-sections are very similar, although small differences are present. The highest yield stress values predicted at the downstream cross-section, are slightly higher than for the upstream cross-section (see also Figure 6). These differences have their impact on the post-yield localization, i.e. necking, as will be explained with more detail in the next subsection.

To indicate the significant influence of the mold temperature on $S_{a}$ and as a consequence also on the yield stress values, computed values for the true yield stress are compared for three different mold temperatures, i.e. $30^{\circ} \mathrm{C}, 90^{\circ} \mathrm{C}$, and $130{ }^{\circ} \mathrm{C}$. Figure 6 shows the results over both the center height and center width of the ASTM tensile bars (see inset) at three different cross-sections and mold temperatures. As expected, higher mold temperatures give higher values for the 


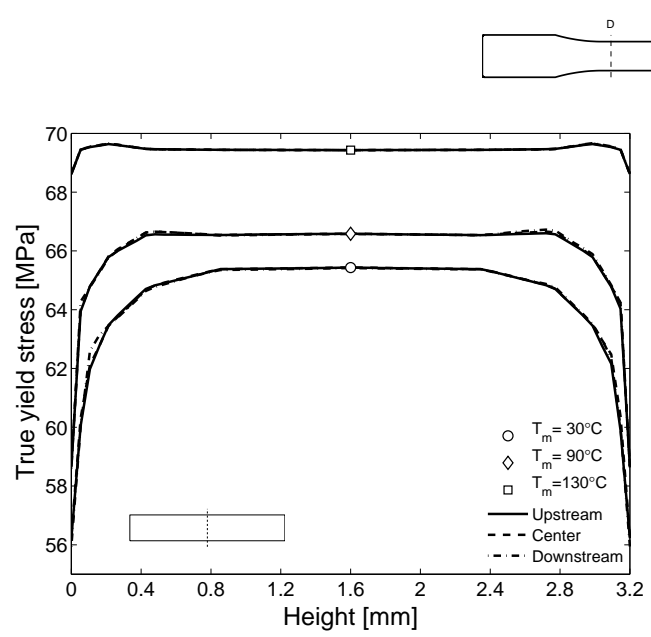

(a)

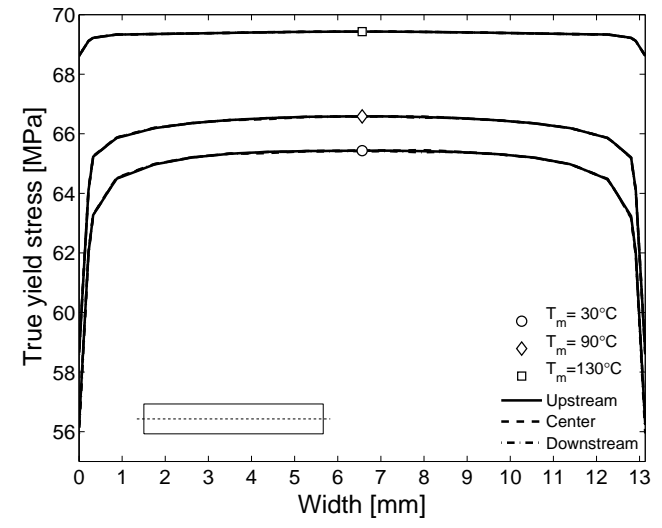

(b)

Figure 6: Computed true yield stress distribution at $\dot{\varepsilon}=10^{-3} \mathrm{~s}^{-1}$ for three mold temperatures and three cross-sections. $(a)$ : Center height of the ASTM tensile bar. (b): Center width of the ASTM tensile bar.

yield stress. Furthermore, differences between the highest and lowest yield stress values reduce as mold temperature increases. The results also show that the effect of the intersecting cooling profiles is more pronounced for the higher mold temperatures. In fact, for the lowest mold temperature of $30{ }^{\circ} \mathrm{C}$ this effect is even absent. One can also observe that this particular effect, although possibly an artifact of the numerical method, is only visible over the center height of the injection molded ASTM tensile bar (Figure 6(a)), and is not present over the center width (Figure 6(b)). For clarity, numerical values of the previous shown results are given in Table 4. The average values for $S_{a}$ and $\sigma_{y}$ are volume-weighted averages over the straight gauge section of the ASTM D638 type I tensile bar. 


\section{Macroscopic Properties}

In order to be able to compare the numerical results with experimental data, the

calculated nodal $S_{a}$-values are transferred to the structural analysis mesh. As a result, structural analysis simulations can be performed with a thermodynamic state parameter distribution along the complete component. Experimental and numerical results of uniaxial tensile tests performed on injection molded ASTM D638 type I test samples with different mold temperatures are shown in Figure 7(a). The model prediction with a distributed thermodynamic state parameter is able to very satisfactorily capture the experimental macroscopic stress-strain response, even necking. The engineering strains between approximately $\varepsilon=0.02$ and $\varepsilon=0.05$ are somewhat overpredicted by the model, which is due to the fact that the linear relaxation spectrum is determined on a different grade of polycarbonate, i.e. PC Lexan $101 \mathrm{R}^{33}$, and, furthermore, it is determined on uniaxial compression tests, instead of uniaxial tensile tests. This slight overprediction, although less pronounced, of the initial viscoelastic regime is also visible in Figure 1, which shows experimental data of PC Lexan $121 \mathrm{R}^{10,33}$ and model predictions using the relaxation spectrum determined on PC Lexan $101 \mathrm{R}^{33}$.

If the distributed thermodynamic state simulation at $T_{\text {mold }}=90{ }^{\circ} \mathrm{C}$ is compared with the prediction of a constant $S_{a}$ for the whole component, i.e. $S_{a}=32.0$ (see Table 4), it is seen in Figure 7(b) that the experimental data are captured equally well up to yield. However, necking is predicted less strong for the latter case, although still satisfactorily. Simulations also reveal that post-yield localization for a constant thermodynamic state parameter along the tensile bar appears in the center cross-section of the component. On the contrary, for a distributed 


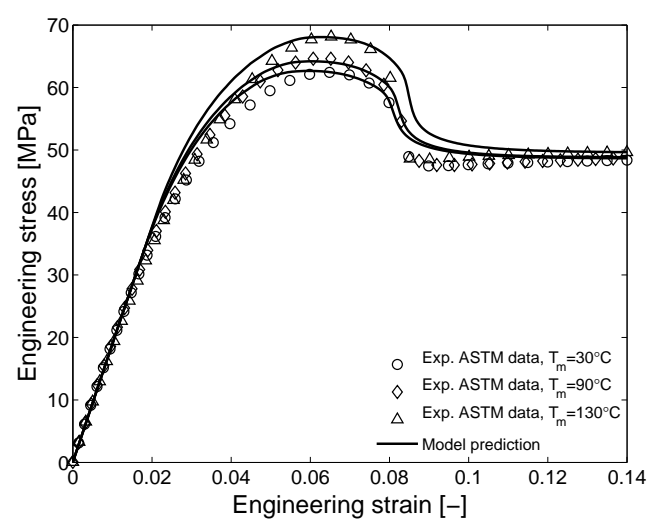

(a)

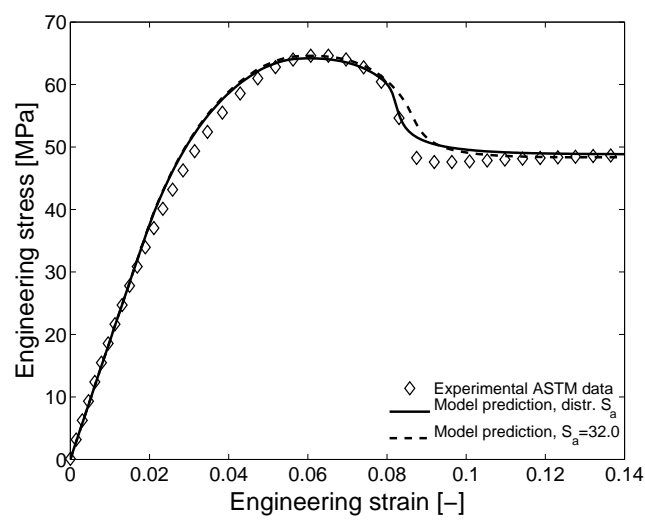

(b)

Figure 7: (a) Experimental data and numerical prediction of a uniaxial tensile test performed at room temperature and at a constant linear strain rate of $10^{-2} \mathrm{~s}^{-1}$ for ASTM samples with mold temperatures of $30{ }^{\circ} \mathrm{C}, 90{ }^{\circ} \mathrm{C}$, and $130{ }^{\circ} \mathrm{C}$. (b) Difference between model predictions with a distributed thermodynamic state parameter $S_{a}$ and a constant $S_{a}$ for $T_{\text {mold }}=90^{\circ} \mathrm{C}$.

$S_{a}$ necking occurs always closer to one of the test set-up clamps, similar to what is seen in experiments. If this takes place either in an upstream or downstream cross-section, turns out to depend on the mold temperature. This leads us to the conclusion that the location of the necking depends, amongst others, on differences in material properties. Of course, it is not the only reason for the location of the neck appearance. Differences in temperature profiles during cooling also lead to differences in dimensions. Furthermore, small surface imperfections, e.g. due to handling or a scratch on the mold surface, provoke small concentrations of stresses. These effects, which can eventually force the initiation of a neck, are not taken into account in the numerical method.

Uniaxial tensile test experiments at different strain rates and different test temperatures were conducted on ASTM D638 type I tensile bars, injection molded at 


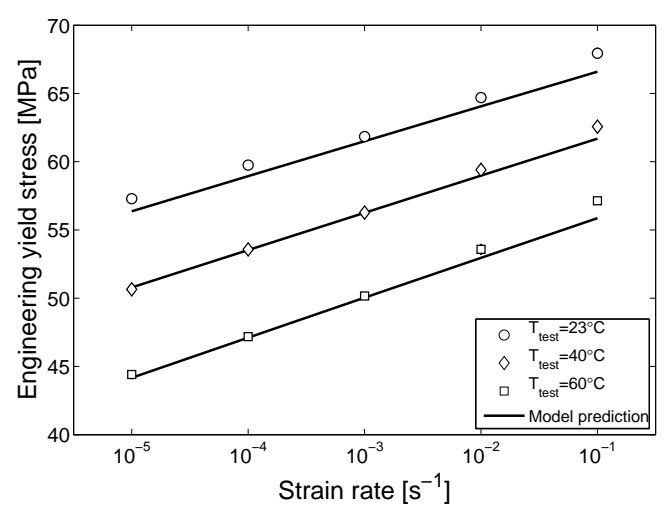

(a)

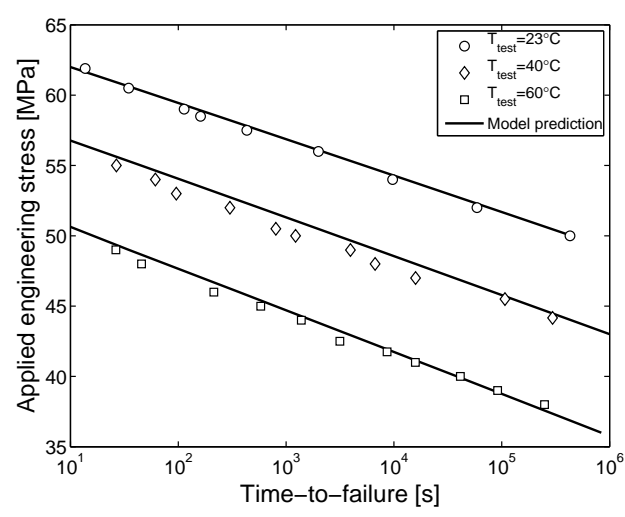

(b)

Figure 8: $(a)$ : Engineering yield stress as a function of strain rate. $(b)$ : Time-tofailure as a function of applied engineering stress. Experiments are conducted at three test temperatures $T_{\text {test }}$ on ASTM D638 type I samples injection molded with $T_{\text {mold }}=90^{\circ} \mathrm{C}$.

$T_{\text {mold }}=90{ }^{\circ} \mathrm{C}$. Results are shown in Figure 8(a). Model predictions are in excellent agreement with experimental results. To verify the prediction of the long-term behavior, creep experiments were conducted and the results are compared with the numerical outcome, as shown in Figure 8(b). Again, excellent agreement is accomplished. Hence, once the deformation and aging kinetics of the material are adequately determined, this modeling approach is able to predict both short- as well as long-term behavior at a range of temperatures.

In the previous subsection, numerical results already showed that different mold temperatures lead to different distributions of the thermodynamic state parameter. To check whether the followed numerical approach gives quantitative results over a range of injection molding conditions, uniaxial tensile experiments at $\dot{\varepsilon}=10^{-2} \mathrm{~s}^{-1}$ were performed on ASTM bars injection molded with mold temperature ranging from $T_{\text {mold }}=30{ }^{\circ} \mathrm{C}$ to $T_{\text {mold }}=130{ }^{\circ} \mathrm{C}$. Comparison between 


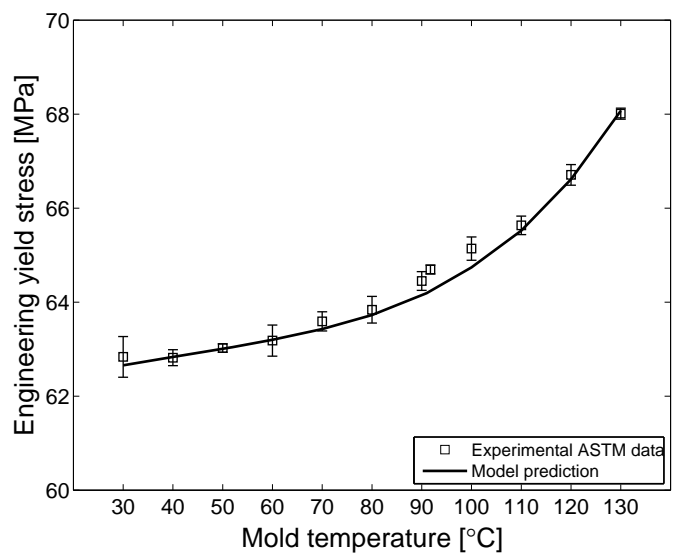

Figure 9: Engineering yield stress as a function of mold temperature measured at a constant linear strain rate of $10^{-2} \mathrm{~s}^{-1}$ and room temperature for an ASTM D638 type I test sample.

experimental data and simulation results are shown in Figure 9. An excellent quantitative agreement is demonstrated by the numerical method, generally showing a prediction within the small range of experimental error or occasionally a slight underprediction.

As a last validation for the applied method, it was checked how well the influence of sample thickness could be predicted. For this, tensile bars were fabricated from injection molded square plates at $T_{\text {mold }}=90{ }^{\circ} \mathrm{C}$ with thicknesses ranging from $1 \mathrm{~mm}$ to $4 \mathrm{~mm}$ by a precision machining operation. Uniaxial tensile tests were performed at $\dot{\varepsilon}=10^{-3} \mathrm{~s}^{-1}$. Comparison between experimental data and simulation results are shown in Figure 10, where the experimental data for the $1 \mathrm{~mm}$ and $4 \mathrm{~mm}$ plates have already been published previously ${ }^{26,27}$. The model predicts the influence of the sample thickness as shown by the experimental data quite well. Though, contrary to results for the injection molded ASTM tensile bars, the model has a tendency to overestimate the experimental data for the case 


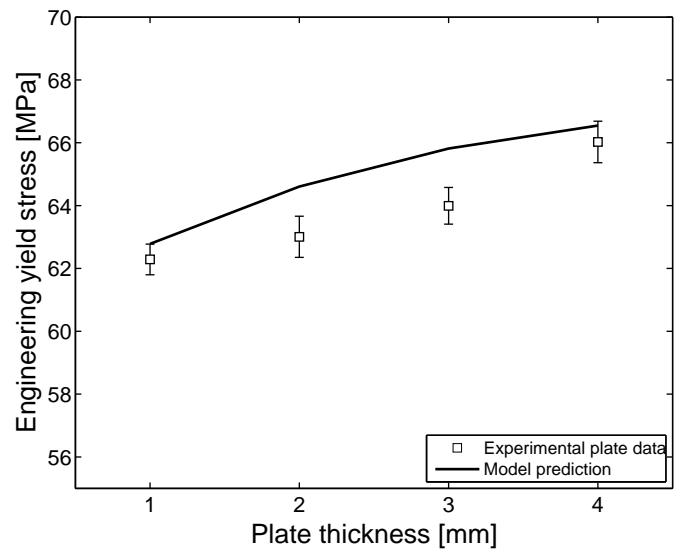

Figure 10: Engineering yield stress as a function of sample thickness measured at a constant linear strain rate of $10^{-3} \mathrm{~s}^{-1}$ and room temperature for a precision machined tensile bar fabricated from a square plate. $T_{\text {mold }}=90{ }^{\circ} \mathrm{C}$.

of samples fabricated from square plates. It is suggested that this is due to the sample preparation by means of a machining operation, that is believed to influence the thermodynamic state of the surface material ${ }^{30}$. A local rejuvenation of the surface material would reduce the measured engineering yield stresses. A minor but uncontrollable change of the thermodynamic state of surface material due to machining is also supported by the fact that the standard deviation of the experimental data is higher than for the measurements on the injection molded ASTM tensile bars.

Nevertheless, even predictions of the precision machined tensile bar experimental data are still very satisfactorily, with only a maximum overestimation of 1.8 $\mathrm{MPa}$ for the $3 \mathrm{~mm}$ plate, i.e. $\sim 2.5 \%$. Since a yield stress distribution is used for the structural analysis simulations, the influence of the machining operation could also be taken into account. However, it should then be investigated to what depth and to what extent a milling operation influences the surface material, which 
is beyond the scope of the current investigation.

\section{Conclusions}

A numerical methodology is presented that takes into account a distribution of mechanical properties, i.e. yield stresses, along the geometrical dimensions of injection molded products, as a consequence of the inhomogeneous thermo-mechanical history experienced during the processing phase, for the structural analysis of polycarbonate products. The present methodology is based on a previously developed modeling approach ${ }^{27,30}$ that incorporates both deformation as well as aging kinetics.

As a first step, the deformation kinetics of the material under investigation has been determined by measuring the large strain intrinsic behavior with "frictionfree" uniaxial compression tests at various strain rates and testing temperatures. Next, the material's aging kinetics are characterized by measuring the yield stress evolution as a function of aging time at several annealing temperatures below $T_{g}$. Model parameters for the material's constitutive equation are determined from these experimental characterization data. Finally, the local thermodynamic state of a material element, i.e. a variable directly related to the material's yield stress, is calculated from the temperature profile as a result from the injection molding process, and transferred to nodal values of a finite element mesh for the structural analysis of a polycarbonate component.

Comparison of simulated results with experimental data of uniaxial tensile tests at a range of strain rates and temperatures performed on injection molded tensile bars and rectangular plates showed an excellent quantitative agreement. 
Even the significant influence of mold temperatures and component wall thickness on the macroscopic properties was predicted correctly. Furthermore, creep experiments with various applied stresses were also quantitatively predicted. The results also indicated that the (strong) localization, i.e. necking, generally observed in uniaxial tensile experiments, is triggered, amongst others, by the distribution of the intrinsic properties.

From an engineering point of view and once a material is properly characterized, this methodology can provide a powerful tool for polymer product and mold designers to yield a correct, safe, and sustainable component design. It will allow them to gain insight into the consequences of specific design choices of e.g. the location of cooling channels in the mold or the effects of hot spots on the service life performance of complex-shaped products in a very early design stage. Furthermore, not only the more "traditional" process variables, such as injection temperature and flow rate or amount and location of the injection molding gates, can be taken into consideration, but also more specific process parameters as the mold temperature or flux and temperature of the cooling medium used in the molding process.

Acknowledgements: Financial support from the Spanish Government (Ministry of Science and Innovation, Ministry of Economy and Competitiveness) through grant numbers RYC-2010-07171 and DPI2011-25470 is gratefully acknowledged.

\section{References}

[1] Niklas H and Kausch von Schmeling HH. Kunststoffe 53: 886-891 (1963). 
[2] Narisawa I, Ishikawa M and Ogawa H. J Polym Sci Polym Phys Ed 16: 1459-1470 (1978).

[3] van Melick HGH, Govaert LE and Meijer HEH. Polymer 44: 3579-3592 (2003).

[4] Boyce MC, Parks DM and Argon AS. Mech Mater 7: 15-33 (1988).

[5] Boyce MC, Arruda EM and Jayachandran R. Polym Eng Sci 34: 716-725 (1994).

[6] Wu PD and van der Giessen E. J Mech Phys Solids 41: 427-456 (1993).

[7] Buckley CP and Jones DC. Polymer 36: 3301-3312 (1995).

[8] Meijer HEH and Govaert LE. Macromol Chem Phys 204: 274-288 (2003).

[9] Wu JJ and Buckley CP. J Polym Sci Part B: Polym Phys 42: 2027-2040 (2004).

[10] Klompen ETJ, Engels TAP, Govaert LE and Meijer HEH. Macromolecules 38: $6997-7008$ (2005).

[11] Boyce MC and Arruda EM. Polym Eng Sci 30: 1288-1298 (1990).

[12] G'Sell C, Hiver JM, Dahoun A and Souahi A. J Mater Sci 27: 5031-5039 (1992).

[13] G'Sell C, Hiver JM and Dahoun A. Int J Solid Struct 39: 3857-3872 (2002).

[14] Struik LCE. Physical Aging in Amorphous Polymners and Other Materials. Elsevier, Amsterdam (1978). 
[15] Hutchinson JM. Prog Polym Sci 20: 703-760 (1995).

[16] Popli R and Mandelkern L. J Polym Sci Part B: Polym Phys 25: 441-483 (1987).

[17] Schrauwen BAG, Janssen RPM, Govaert LE and Meijer HEH. Macromolecules 37: 6069-6078 (2004).

[18] van Erp TB, Govaert LE and Peters GWM. Macromol Mater Eng 298: 348358 (2013).

[19] Senden DJA, Peters GWM, Govaert LE and van Dommelen JAW. Polymer 54: 5899-5908 (2013).

[20] Cross A and Haward RN. Polymer 19: 677-682 (1978).

[21] Struik LCE. Polymer 30: 815-830 (1989).

[22] Bauwens-Crowet C and Bauwens JC. Polymer 23: 1599-1604 (1982).

[23] Narayanaswamy OS. J Am Ceram Soc 54: 491-498 (1971).

[24] DeBolt MA, Easteal AJ, Macedo PB and Moynihan CT. J Am Ceram Soc 59: 16-21 (1976).

[25] Kovacs AJ, Aklonis JJ, Hutchinson JM and Ramos AR. J Polym Sci Polym Phys Ed 17: 1097-1162 (1979).

[26] Engels TAP, Govaert LE, Peters GWM and Meijer HEH. J Polym Sci Part B 44: 1212-1225 (2006). 
[27] Govaert LE, Engels TAP, Klompen ETJ, Peters GWM and Meijer HEH. Int Polym Process 20: 170-177 (2005).

[28] Klompen ETJ, Engels TAP, van Breemen LCA, Schreurs PJG, Govaert LE and Meijer HEH. Macromolecules 38: 7009-7017 (2005).

[29] Janssen RPM, de Kanter D, Govaert LE and Meijer HEH. Macromolecules 41: $2520-2530$ (2008).

[30] Engels TAP, van Breemen LCA, Govaert LE and Meijer HEH. Macromol Mater Eng 294: 829-838 (2009).

[31] Engels TAP, Schrauwen BAG, van Breemen LCA and Govaert LE. Int Polym Process 24: 167-173 (2009).

[32] Tervoort TA, Klompen ETJ and Govaert LE. J Rheol 40: 779-797 (1996).

[33] van Breemen LCA, Klompen ETJ, Govaert LE and Meijer HEH. J Mech Phys Solids 59: 2191-2207 (2011).

[34] Haward RN and Thackray G. Proc Royal Soc London, Series A: Math, Phys Eng Sci 302: 453-472 (1968).

[35] Visser HA, Bor TC, Wolters M, Engels TAP and Govaert LE. Macromol Mater Eng 295: 637-651 (2010).

[36] McKenna GB. Comprehensive Polymer Science, Vol. 2 Polymer Properties, Pergamon Press, Oxford, chap. Glass Formation and Glassy Behavior, pp. 311-362 (1989). 


\section{List of Tables}

Table 1: Model parameters for polycarbonate ${ }^{10,35}$ as used for the EGP-model .

\begin{tabular}{|c|c|c|c|c|c|c|c|c|}
\hline $\begin{array}{c}G_{r} \\
{[\mathrm{MPa}]}\end{array}$ & $\begin{array}{c}\kappa \\
{[\mathrm{MPa}]}\end{array}$ & $\begin{array}{c}\Delta U_{y} \\
{[\mathrm{~kJ} / \mathrm{mol}]}\end{array}$ & $\begin{array}{c}T_{r e f} \\
{[\mathrm{~K}]}\end{array}$ & $\begin{array}{c}\mu \\
{[-]}\end{array}$ & $\begin{array}{c}V_{y}^{*} \\
{\left[\mathrm{~m}^{3} / \mathrm{mol}\right]}\end{array}$ & $\begin{array}{c}r_{0} \\
{[-]}\end{array}$ & $\begin{array}{c}r_{1} \\
{[-]}\end{array}$ & $\begin{array}{c}r_{2} \\
{[-]}\end{array}$ \\
\hline \hline 26 & 3750 & 327 & 296 & 0.08 & $3.52 \times 10^{-3}$ & 0.965 & 50 & -3 \\
\hline
\end{tabular}

Table 2: Reference Maxwell relaxation spectrum for polycarbonate ${ }^{33}$ as used for the EGP-model .

\begin{tabular}{|c|l|c|c|}
\hline Mode $i$ & $\begin{array}{c}\eta_{0, i, r e f} \\
{[\mathrm{MPa} \cdot \mathrm{s}]}\end{array}$ & $\begin{array}{c}G_{i} \\
{[\mathrm{MPa}]}\end{array}$ & $\begin{array}{c}\lambda_{i} \\
{[\mathrm{~s}]}\end{array}$ \\
\hline \hline 1 & $2.10 \times 10^{11}$ & $3.52 \times 10^{2}$ & $5.97 \times 10^{8}$ \\
2 & $3.48 \times 10^{9}$ & $5.55 \times 10^{1}$ & $6.27 \times 10^{7}$ \\
3 & $2.95 \times 10^{8}$ & $4.48 \times 10^{1}$ & $6.58 \times 10^{6}$ \\
4 & $2.84 \times 10^{7}$ & $4.12 \times 10^{1}$ & $6.89 \times 10^{5}$ \\
5 & $2.54 \times 10^{6}$ & $3.50 \times 10^{1}$ & $7.26 \times 10^{4}$ \\
6 & $2.44 \times 10^{5}$ & $3.20 \times 10^{1}$ & $7.63 \times 10^{3}$ \\
7 & $2.20 \times 10^{4}$ & $2.75 \times 10^{1}$ & $8.00 \times 10^{2}$ \\
8 & $2.04 \times 10^{3}$ & $2.43 \times 10^{1}$ & $8.40 \times 10^{1}$ \\
9 & $1.83 \times 10^{2}$ & $2.07 \times 10^{1}$ & $8.84 \times 10^{0}$ \\
10 & $1.68 \times 10^{1}$ & $1.81 \times 10^{1}$ & $8.28 \times 10^{-1}$ \\
11 & $1.51 \times 10^{0}$ & $1.54 \times 10^{1}$ & $9.81 \times 10^{-2}$ \\
12 & $1.40 \times 10^{-1}$ & $1.36 \times 10^{1}$ & $1.03 \times 10^{-2}$ \\
\hline
\end{tabular}

Table 3: Model parameters for the aging kinetics of polycarbonate ${ }^{10}$ as used for the EGP-model.

\begin{tabular}{|c|c|c|c|c|}
\hline $\begin{array}{c}c_{0} \\
{[-]}\end{array}$ & $\begin{array}{c}c_{1} \\
{[-]}\end{array}$ & $\begin{array}{c}t_{0} \\
{[\mathrm{~s}]}\end{array}$ & $\begin{array}{c}\Delta U_{a} \\
{[\mathrm{~kJ} / \mathrm{mol}]}\end{array}$ & $\begin{array}{c}T_{r e f} \\
{[\mathrm{~K}]}\end{array}$ \\
\hline \hline-4.41 & 3.30 & 1 & 205 & 296 \\
\hline
\end{tabular}


Table 4: Minimum, maximum, and volume-weighted average true yield stress and thermodynamic state parameter values in the gauge section of ASTM tensile bars for three different mold temperatures.

\begin{tabular}{|c||c|c|c|c|}
\hline $\begin{array}{c}T_{\text {mold }} \\
{\left[{ }^{\circ} \mathrm{C}\right]}\end{array}$ & $\begin{array}{c}\sigma_{y, \text { min }} \\
{[\mathrm{MPa}]}\end{array}$ & $\begin{array}{c}\sigma_{y, \text { max }} \\
{[\mathrm{MPa}]}\end{array}$ & $\begin{array}{c}\sigma_{y, \text { ave }} \\
{[\mathrm{MPa}]}\end{array}$ & $\begin{array}{c}S_{a, \text { ave }} \\
{[-]}\end{array}$ \\
\hline \hline 30 & 55.9 & 65.5 & 64.0 & 30.6 \\
90 & 58.6 & 66.8 & 65.7 & 32.0 \\
130 & 68.6 & 69.7 & 69.4 & 35.2 \\
\hline
\end{tabular}




\section{List of Figures}

1 Intrinsic stress-strain response of polycarbonate in uniaxial compression tests. (a) Stress-strain response at different strain rates and an initial thermodynamic state of $S_{a}=27.5 ;(b)$ Stress-strain response with different thermal histories (i.e. quenched and annealed) at a strain rate of $10^{-3} \mathrm{~s}^{-1}$. Symbols are experimental data; solid lines are model predictions; dashed line is the completely rejuvenated state. Experimental data are taken from Klompen et al. ${ }^{10}$ and Van Breemen et al. ${ }^{33}$. . . . . . . . . 10

2 (a) Evolution of the uniaxial tensile yield stress at a strain rate of $10^{-2} \mathrm{~s}^{-1}$ as a function of annealing time for different annealing temperatures. (b) Master curve for a reference annealing temperature of $80{ }^{\circ} \mathrm{C}$ constructed from the experimental data by using time-temperature superposition. Symbols are experimental data; solid lines are model predictions. Experimental data are taken from Klompen et al. ${ }^{10} \ldots \ldots \ldots \ldots \ldots$

3 Injection molded ASTM tensile bars $(a)$, plate $(b)$, and tensile bars made thereof $(c) \ldots \ldots \ldots \ldots \ldots$

4 (a): Cooling profiles over the height at the center of the injection molded ASTM tensile bar with $T_{\text {mold }}=90^{\circ} \mathrm{C}$. Horizontal dashed line: glass transition temperature $T_{g}$. (b): Temperature versus time of these profiles from the moment the temperature drops below $T_{g} \ldots \ldots \ldots \ldots \ldots \ldots$ 
5 Predicted true yield stress distribution at $\dot{\varepsilon}=10^{-3} \mathrm{~s}^{-1}$ for a mold temperature of $T_{\text {mold }}=90{ }^{\circ} \mathrm{C}:(a)$ cross-section downstream from the injection point, $(b)$ cross-section at the center of the tensile bar, and $(c)$ cross-section upstream from the injection point. . . . . 18

6 Computed true yield stress distribution at $\dot{\varepsilon}=10^{-3} \mathrm{~s}^{-1}$ for three mold temperatures and three cross-sections. $(a)$ : Center height of the ASTM tensile bar. $(b)$ : Center width of the ASTM tensile bar.

7 (a) Experimental data and numerical prediction of a uniaxial tensile test performed at room temperature and at a constant linear strain rate of $10^{-2} \mathrm{~s}^{-1}$ for ASTM samples with mold temperatures of $30{ }^{\circ} \mathrm{C}, 90{ }^{\circ} \mathrm{C}$, and $130{ }^{\circ} \mathrm{C}$. (b) Difference between model predictions with a distributed thermodynamic state parameter $S_{a}$ and a constant $S_{a}$ for $T_{\text {mold }}=90{ }^{\circ} \mathrm{C} \ldots \ldots \ldots \ldots . \ldots \ldots$

8 (a): Engineering yield stress as a function of strain rate. $(b)$ : Time-to-failure as a function of applied engineering stress. Experiments are conducted at three test temperatures $T_{\text {test }}$ on ASTM D638 type I samples injection molded with $T_{\text {mold }}=90{ }^{\circ} \mathrm{C} . \ldots .23$

9 Engineering yield stress as a function of mold temperature measured at a constant linear strain rate of $10^{-2} \mathrm{~s}^{-1}$ and room temperature for an ASTM D638 type I test sample. . . . . . . . . . . . . 24

10 Engineering yield stress as a function of sample thickness measured at a constant linear strain rate of $10^{-3} \mathrm{~s}^{-1}$ and room temperature for a precision machined tensile bar fabricated from a square plate $T_{\text {mold }}=90{ }^{\circ} \mathrm{C} \ldots \ldots \ldots \ldots \ldots \ldots$ 


\section{Graphical Table of Contents}

\section{Yield Stress Distribution in Injection-Molded Glassy Polymers}

Wilco M.H. Verbeeten*, Marc J.W. Kanters, Tom A.P. Engels and Leon E. Govaert

A methodology for structural analysis simulations is presented that incorporates the distribution of mechanical properties due to injection molding processes along the geometrical dimensions of amorphous polymer products.

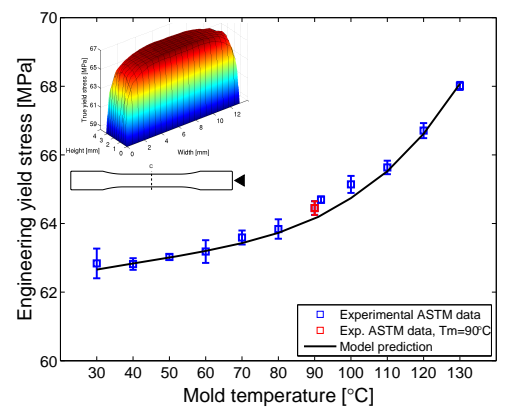

